\title{
BMJ Open Motivation and main flavour of use, use with nicotine and dual use of electronic cigarettes in Barcelona, Spain: a cross- sectional study
}

\author{
Kailey Bunch, ${ }^{1}$ Marcela Fu, ${ }^{2,3,4}$ Montse Ballbè, ${ }^{2,3,5}$ Nuria Matilla-Santader, ${ }^{1}$ \\ Cristina Lidón-Moyano, ${ }^{1}$ Juan Carlos Martin-Sanchez, ${ }^{1}$ Esteve Fernandez, ${ }^{2,3,4}$ \\ Jose M Martínez-Sánchez ${ }^{1,2,3}$
}

To cite: Bunch K, Fu M, Ballbè M, et al. Motivation and main flavour of use, use with nicotine and dual use of electronic cigarettes in Barcelona, Spain: a crosssectional study. BMJ Open 2018;8:e018329. doi:10.1136/ bmjopen-2017-018329

- Prepublication history for this paper is available online. To view these files, please visit the journal online (http://dx.doi. org/10.1136/bmjopen-2017018329).

Received 20 June 2017 Revised 14 December 2017 Accepted 5 February 2018

Check for updates

${ }^{1}$ Group of Evaluation of Health Determinants and Health Policies, Universitat Internacional de Catalunya, Sant Cugat del Valles, Spain

${ }^{2}$ Tobacco Control Unit, Cancer

Prevention and Control Program, Institut Català d'Oncologia,

Barcelona, Spain

${ }^{3}$ Cancer Prevention and Control Group, Institut d'Investigació Biomèdica de Bellvitge-IDIBELL,

Barcelona, Spain

${ }^{4}$ Department of Clinical Sciences, School of Medicine, Universitat de Barcelona, Barcelona, Spain

${ }^{5}$ Addictions Unit, Institute of Neurosciences, Hospital Clínic de Barcelona, Barcelona, Spain

Correspondence to

Dr Jose M Martínez-Sánchez; jmmartinez@uic.es

\section{ABSTRACT}

Objective This study seeks to describe current e-cigarette users' patterns of use including primary motivation for use, dual use, use with nicotine and principal flavour used, according to individuals' sociodemographic characteristics and conventional tobacco consumption in Barcelona, Spain.

Design, setting and participants This is a crosssectional study of adult $(\geq 18)$ current e-cigarette users of Barcelona $(n=600)$, recruited in 2015. Researchers calculated percentages and ORs with their $95 \% \mathrm{Cls}$ of users' e-cigarette use with nicotine, primary motivation for use and preferred flavour. The analyses were stratified by sociodemographic variables including sex, age and level of education and by patterns of e-cigarette use and past and current conventional tobacco use.

Results The most prevalent motivation for using e-cigarettes was to reduce tobacco smoking $(48 \%$, $\mathrm{n}=288)$, followed by quitting smoking $(39.2 \%, \mathrm{n}=235)$, and to use e-cigarettes in places where tobacco smoking was prohibited $(10.2 \%, n=61)$. The most prevalent primary flavour used was tobacco $(49 \%, n=294)$, followed by fruit $(19.5 \%, n=117)$. People younger than 25 years old $(\mathrm{OR}=4.36,95 \% \mathrm{Cl} 1.99$ to 9.57$)$ were more likely than older people to use them where smoking cigarettes was prohibited. A greater proportion of those in the youngest age group preferred fruit flavour compared with older individuals $(29.3 \%, n=41)$.

Conclusions Younger users of e-cigarettes and nonsmokers are more likely to use flavours other than tobacco and to use e-cigarettes mainly for recreational purposes.

\section{INTRODUCTION}

Since the invention of electronic cigarettes (e-cigarettes) in 2003, the prevalence of their awareness and use has significantly increased throughout the world. In the USA during 2010-2013, their awareness increased from $40.9 \%$ to $79.7 \%$, ever use from $3.3 \%$ to $8.5 \%$ and current use from $1.0 \%$ to $2.6 \%$. $^{1}$ Similar increases have also been documented in other countries, including Great Britain, Poland, Finland, Latvia and Korea. ${ }^{23}$ In Spain
Strengths and limitations of this study

- This is the first study in Spain describing the average time of e-cigarette use, number of cartridges and milligrams of nicotine per cartridge and the only one in Europe, to our best knowledge, which assesses users' preferred flavour and motivation for use, stratified by their patterns of e-cigarette use and past and current tobacco consumption.

- The use of face-to-face questionnaires with trained interviewers strengthens the internal validity of the study.

- The sample was recruited using a non-probabilistic sampling method which could affect the external validity of the study.

- The use of a questionnaire in data collection allows for possible recall and response biases.

- The cross-sectional study design only allows for assessment of associations as opposed to causal relationships.

in 2014, prevalence of ever e-cigarette use was estimated at $6.5 \%$ and current use at $1.6 \%$. $^{4}$

E-cigarettes have created a polarisation among researchers, health professionals and the general population about their useful or harmful effects since their commercialisation. Some researchers and citizens, particularly smokers, e-cigarette users and stakeholders, are advocates of e-cigarettes as a useful tool to quit or reduce tobacco consumption, and some people and organisations suggest them as a harm reduction strategy for smokers. ${ }^{5}$ On the other hand, most tobacco control researchers, professionals and activists view e-cigarettes as a pathway to the renormalisation of tobacco products in public and in workplaces, creating new potential nicotine addiction among young people, including those who have never used tobacco. ${ }^{6}$

The current evidence for and against e-cigarettes as a useful tool is contradictory. Some 
studies show that e-cigarettes can be an effective tool for cessation or reduction of tobacco consumption and for the improvement of tobacco-related disease conditions, ${ }^{7-13}$ while a recent systematic review and meta-analysis concluded that they have no effect or even a negative effect. ${ }^{14}$ Moreover, there is a theoretical concern that e-cigarettes may harm public health gains in tobacco reduction by renormalising smoking and creating and sustaining nicotine addiction, since their use, sale and marketing are less regulated than tobacco products, ${ }^{15}$ although the evidence currently is scarce. ${ }^{16}$

Since e-cigarettes have only been on the market for a short time, long-term studies of the potential health effects of e-cigarettes do not yet exist. Some short-term studies have found negative pulmonary effects in users such as lung and airway obstruction. ${ }^{17}$ Furthermore, chemicals such as carcinogenic tobacco-specific nitrosamines and carcinogenic carbonyls including formaldehyde and acetaldehyde ${ }^{17-20}$ have been found in e-cigarette liquid. The long-term effects of these chemicals must continue to be evaluated.

Gaps in information on e-cigarette users' patterns and characteristics of use still exist. Monitoring these patterns of use is necessary to inform decisions aimed at minimising the potential negative effects of e-cigarettes and at maximising their potential benefits. Therefore, the objective of this study is to describe current e-cigarette users' patterns of use, including primary motivation for use, dual use, use with nicotine and main flavour used, according to individuals' sociodemographic characteristics and conventional tobacco consumption.

\section{METHODS}

This is a cross-sectional study of a sample of adult $(\geq 18$ years) current e-cigarette users, and residents of Barcelona in Spain $(n=600)$. Due to limited resources and a necessary sample size that was large relative to the prevalence of e-cigarette use in the population, a probabilistic sampling technique was infeasible. Therefore, the technique known as 'consumer panels' was used to enrol e-cigarette users as described previously elsewhere. ${ }^{21}$ Briefly, this technique is often used in market research to recruit users of uncommonly found products. For this study, e-cigarette users were recruited in all neighbourhoods of the city of Barcelona between February and June 2015 by sensors (specifically trained personnel for the recruitment of uncommon product consumers, in this case, e-cigarette users). The sensors walked around Barcelona neighbourhoods and when they identified individuals using or carrying an e-cigarette in public, the interviewees were approached and asked if they were current e-cigarette users and if they would take part in the study. All participants were current e-cigarette users at the moment of the interview, independent of whether they used it daily or occasionally. To recruit the 600 participants, 665 individuals were approached (a rejection rate of $9.7 \%$ ). A brief face-to-face interview was conducted with the individuals who agreed to participate at that point and again in 2016 to be followed up. The sample size for this study was calculated using the formula for simple random samples $\left(\mathrm{Z} \alpha^{*} \mathrm{pq} / \mathrm{e}\right)^{2}$ for an expected prevalence of $50 \%$ $(\mathrm{P}=\mathrm{q}=0.5)$ to yield the maximum sample size and ensure statistical power. A 95\% CI was used $(\mathrm{Z} \alpha / 2=1.96)$ and an absolute error of 0.04 . We used $50 \%$ as the expected prevalence because we did not know the prevalence of the patterns of use among e-cigarette users and this prevalence maximises the sample size.

\section{Study variables}

The principal variables used in this study are outlined below.

\section{Patterns of use}

These variables included the amount of time in months the participant had been using e-cigarettes, use of the e-cigarette with or without nicotine, number of cartridges of e-liquid with nicotine used per week and milligrams of nicotine the cartridges contained.

\section{Motivation for use}

Users were asked about their primary motivation for using e-cigarettes. The possible responses were: 'to quit tobacco smoking', 'to reduce tobacco smoking', 'to smoke in places where tobacco is prohibited' and 'other reasons'. This last category was open ended and the interviewers wrote the literal response of the participants. For the present study, we do not used the open category $(n=16)$ for the motivation for use.

\section{Flavour used}

Participants were asked an open-ended question about the primary flavour they used with their e-cigarette. Answers such as 'tobacco' or any brand of tobacco cigarettes were categorised as 'tobacco'; answers such as 'menthol' and 'mint' were categorised as 'menthol'; flavours such as 'strawberry', 'apple' and 'watermelon' were categorised as 'fruit'; and flavours like 'chocolate', 'coffee' and 'cinnamon' were categorised as 'others'.

We also obtained information about sex, age (categorised as $<25$ years old, 25-44 years old, 45-64 years old and $\geq 65$ years old), educational level (categorised as primary or less (no education up to middle school diploma), secondary or intermediate (high school) and university or more (university degree)), smoking status at the time of data collection and before initiating use of e-cigarettes (non-smokers and smokers), number of cigarettes smoked per day at the time of data collection and before initiating use of e-cigarettes (categorised as $<10$, $10-19, \geq 20)$ and nicotine dependence before initiating use of e-cigarettes using the heavy smoking index ${ }^{22}$ (categorised as low, medium and high). These variables were used as covariates.

This study is part of a project called: 'Patrón de uso, aceptabilidad y percepción de riesgo de los cigarrillos electrónicos: estudio prospectivo de cohortes con biomarcadores'. 


\section{DATA ANALYSIS}

We calculated the proportion of the qualitative variables (motivation for using e-cigarettes, main flavour, and so on) and median and IQR for the quantitative variables due to the skewed distributions of the data. We used $\mathrm{X}^{2}$ test and Fisher's exact test for qualitative variables, Mann-Whitney U test and Kruskal-Wallis test for quantitative variables with independent samples, and Wilcoxon test for quantitative paired samples (number of cigarettes smoked before and during use of e-cigarettes). We also fit separate multivariate logistic regression models to calculate the ORs with their 95\% CIs of e-cigarette users' use with nicotine, motivation for use and flavour used adjusted for sex and age. Analyses were stratified by sex, age, educational level, conventional tobacco use and use of e-cigarette with or without nicotine. Although we used a non-probabilistic sampling method to recruit the e-cigarette users, our sample was representative of current e-cigarette users according to sex and age.$^{23}$ For this reason, analyses were not weighted. The level of statistical significance was set to a two-sided $\mathrm{P}$ value $<0.05$. All analyses were conducted using Stata V.14.0 statistical software.

\section{RESULTS}

Table 1 shows information about the time the participants were using e-cigarettes, use with nicotine, number of cartridges with nicotine used per week and quantity of nicotine of the cartridges in milligrams. Median time using e-cigarettes was 6 months ( $\mathrm{IQR}=3-12$ months) and was significantly greater in men, users aged 65 years and older, people with university education and current non-smokers (table 1). Fifty-six per cent of e-cigarette users used them with nicotine and $86.8 \%$ of the e-cigarette users were past tobacco smokers (before use of e-cigarettes) compared with $65.2 \%$ who were current smokers (dual users) $(\mathrm{P}<0.001$, not shown in table). Use with nicotine was significantly greater among men, people aged 65 years and older, current and past smokers, and those who smoked 20 or more cigarettes per day. Among participants who used e-cigarettes with nicotine $(n=336)$, median number of cartridges with nicotine used per week was 2 ( $\mathrm{IQR}=1-3$ cartridges) and was greater in users aged 65 and older and those who smoked 20 or more cigarettes per day before initiating use of e-cigarettes (table 1).

Of the total sample, the most prevalent motivation for using e-cigarettes was to reduce tobacco smoking (48\%), followed by quitting smoking $(39.2 \%)$, and to use in places where tobacco smoking was prohibited $(10.2 \%)$ (table 2). People younger than 25 years old $(\mathrm{OR}=4.36$, 95\% CI 1.99 to 9.57 ) and women (OR=1.87, 95\% CI 1.02 to 3.43 ) were more likely to state their main motivation for use as to use e-cigarettes where cigarettes were prohibited, while older people were more likely to use them to quit smoking tobacco $(\geq 65, \mathrm{OR}=13.8,95 \%$ CI 2.62 to 72.41) (table 2). Those whose main motivation of e-cigarette use was to quit tobacco smoking had been using e-cigarettes the longest ( $\geq 2$ years, $\mathrm{OR}=10.22,95 \%$ CI 2.57 to 40.62) (table 2). Individuals whose main motivation of use was to use them in places where tobacco smoking was prohibited were more likely to use fruit flavour $(\mathrm{OR}=8.72$, $95 \% \mathrm{CI} 3.76$ to 20.26$)$ and to have been non-smokers before initiating e-cigarette use $(\mathrm{OR}=36.15,95 \% \mathrm{CI}$ 18.36 to 71.18 ) or non-smokers while using e-cigarettes (OR=10.60, 95\% CI 5.40 to 20.82) (table 2).

Of the overall sample, $99 \%$ reported using an e-cigarette flavour. The most prevalent flavour used was tobacco $(49 \%)$, followed by fruit $(19.5 \%)$ (table 3 ). Tobacco flavour was more frequent among men $(\mathrm{OR}=2.13,95 \% \mathrm{CI}$ 1.51 to 3.00$)$ and people aged $25-44(\mathrm{OR}=2.25,95 \%$ CI 0.51 to 9.84 ) (table 3). Fruit flavour was more prevalent among women (women, 23.9\%; men, $11.9 \% ; \mathrm{P}<0.001$ ) and the youngest age group, showing a negative doseresponse pattern with increasing age $(<25,29.3 \%$; 25-44, $17.7 \%$; 45-64, 14.4\%; $\geq 65,12.5 \%$; $\mathrm{P}=0.008$ ) (table 3), although OR did not yield statistically significant results. Users of tobacco flavour smoked currently the greatest number of cigarettes per day $(>20$ cigarettes, $\mathrm{OR}=6.45$, $95 \%$ CI 1.84 to 22.59 ) and were more likely to be past and current smokers (table 3 ).

\section{DISCUSSION}

In our study, we found a relatively short median time that e-cigarette users in Barcelona had been using e-cigarettes. We also found a high proportion $(65 \%)$ of users smoke conventional cigarettes (dual use), and nearly one in seven $(13 \%)$ were non-smokers before beginning use of e-cigarettes. Participants' main motivations for using e-cigarettes were predominantly to quit or reduce tobacco consumption. For use in places where cigarettes are banned (recreational use) was the least common motivation for use, although young people were more likely than older people to use them for this reason. While the most popular flavour was tobacco, more than $50 \%$ of the sample participants used other flavours.

The median time using e-cigarettes of our sample participants was 6 months. A study of European current e-cigarette users conducted in 2014 found a median time of 10 months and the average time of use was significantly longer in former cigarette smokers than current smokers (11 and 8 , respectively). ${ }^{23}$ We found that median time of use was significantly greater in older people. This could be related to their motivations for using e-cigarettes as being much more likely to use to quit smoking cigarettes. If people notice a decrease in the number of cigarettes they smoke since they began using e-cigarettes, there is an incentive for them to continue. Our findings are supported by a study that found higher rates of continued use for those who had goal-oriented reasons for use than for those who had non-goal-oriented reasons. ${ }^{24}$ Longer duration of e-cigarette use has been associated with an increased frequency of use and a simultaneous decrease in tobacco smoking. ${ }^{25}$ On one hand, if a longer duration of e-cigarette use eventually leads to complete tobacco smoking cessation, public health implications may be 


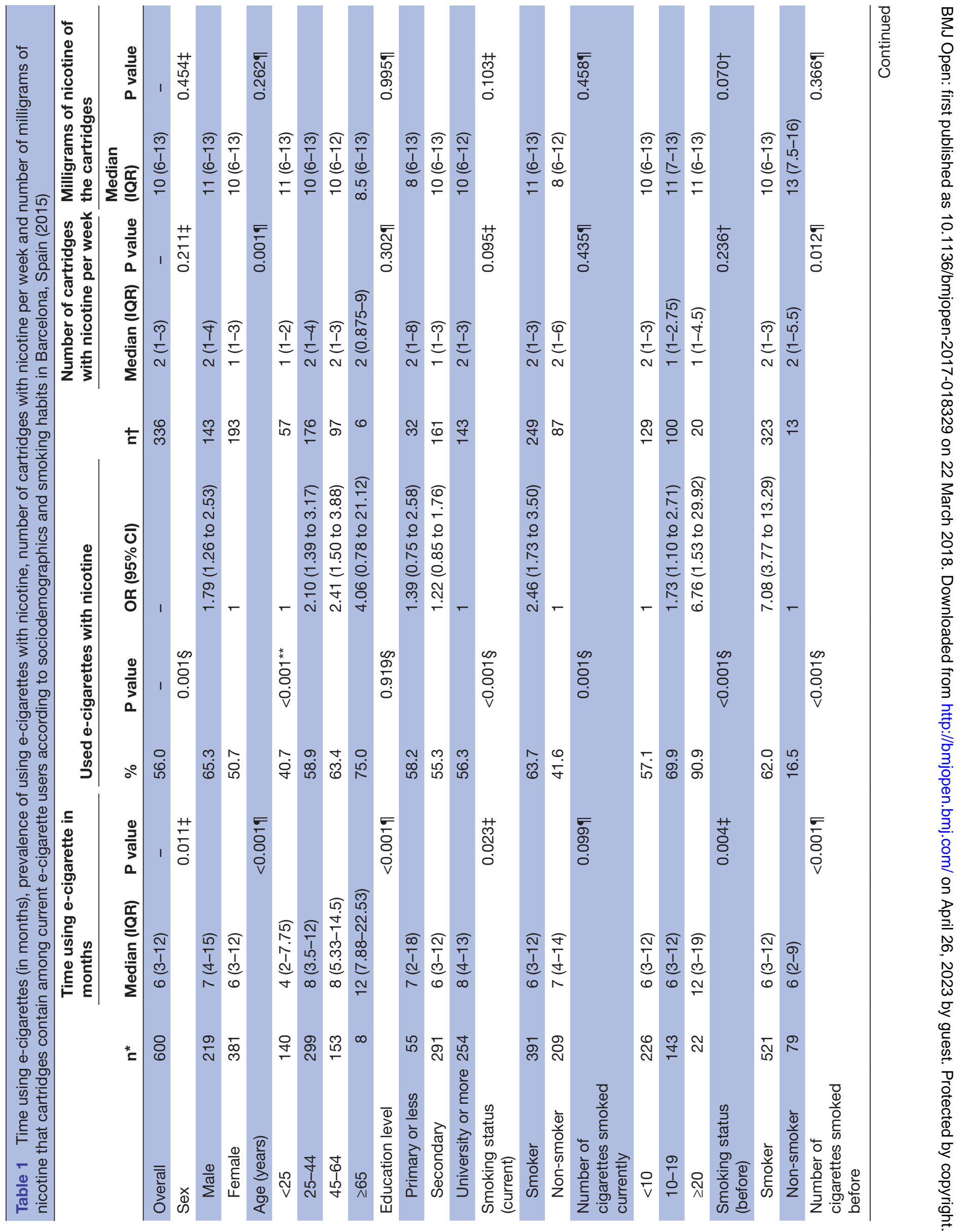




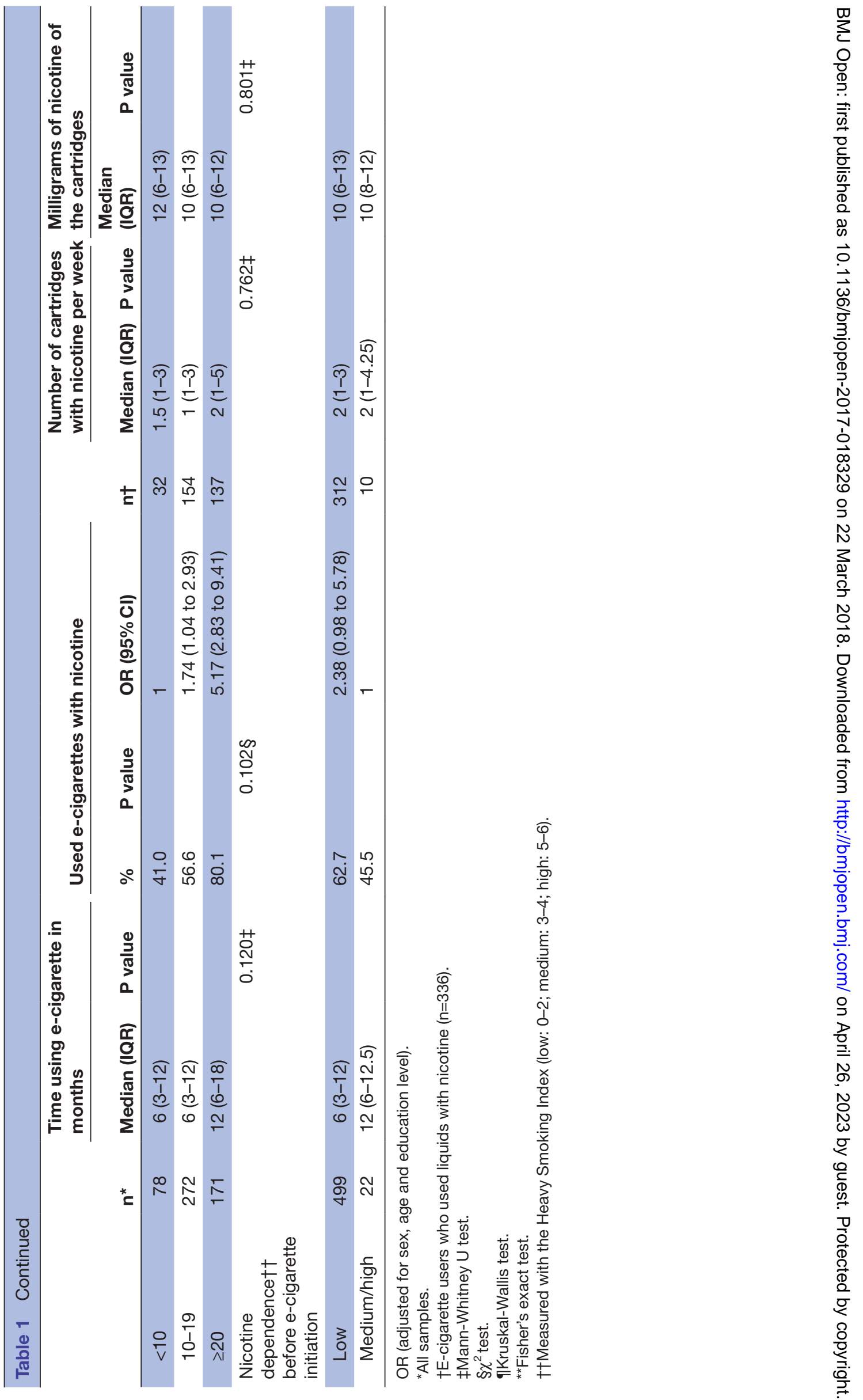




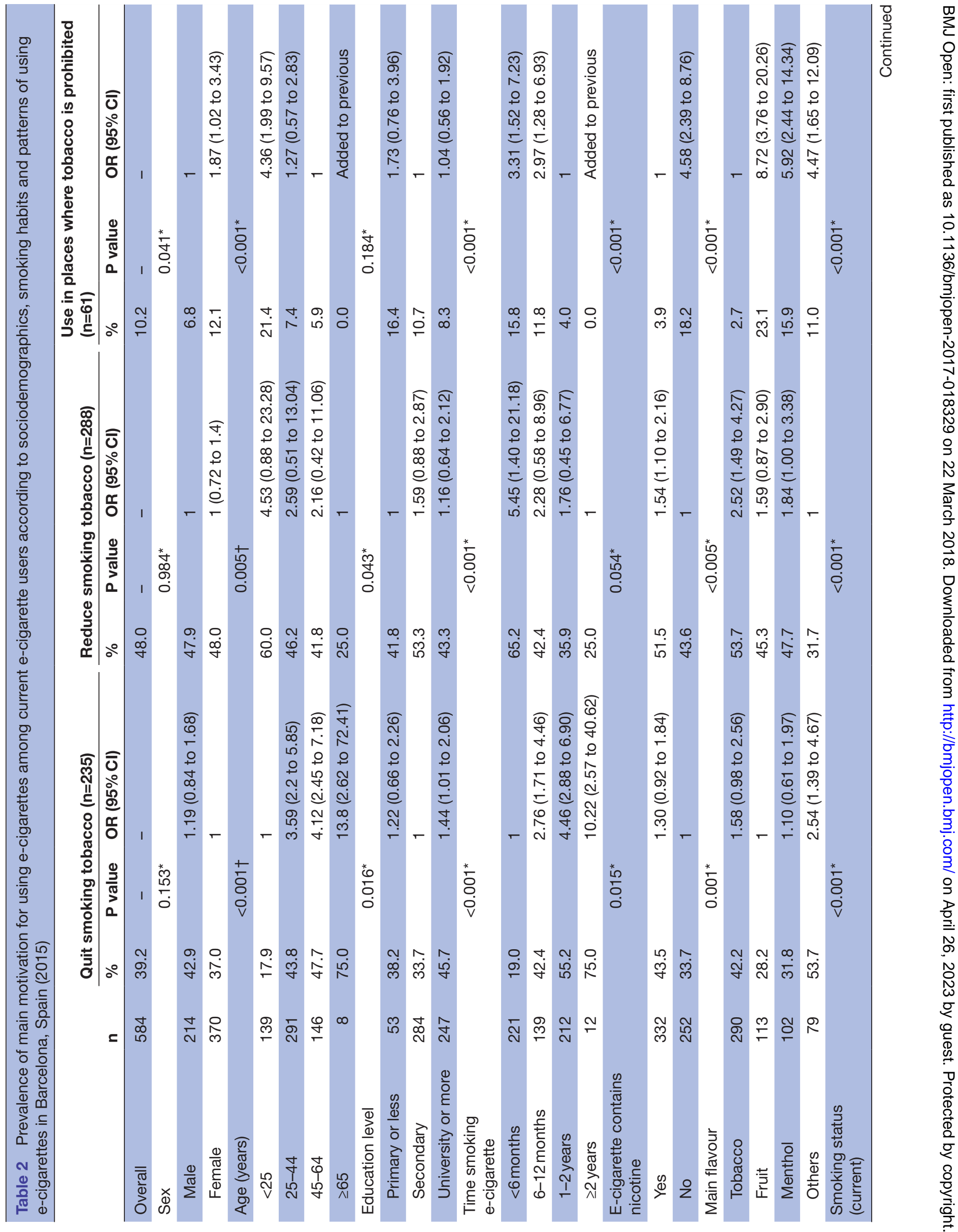




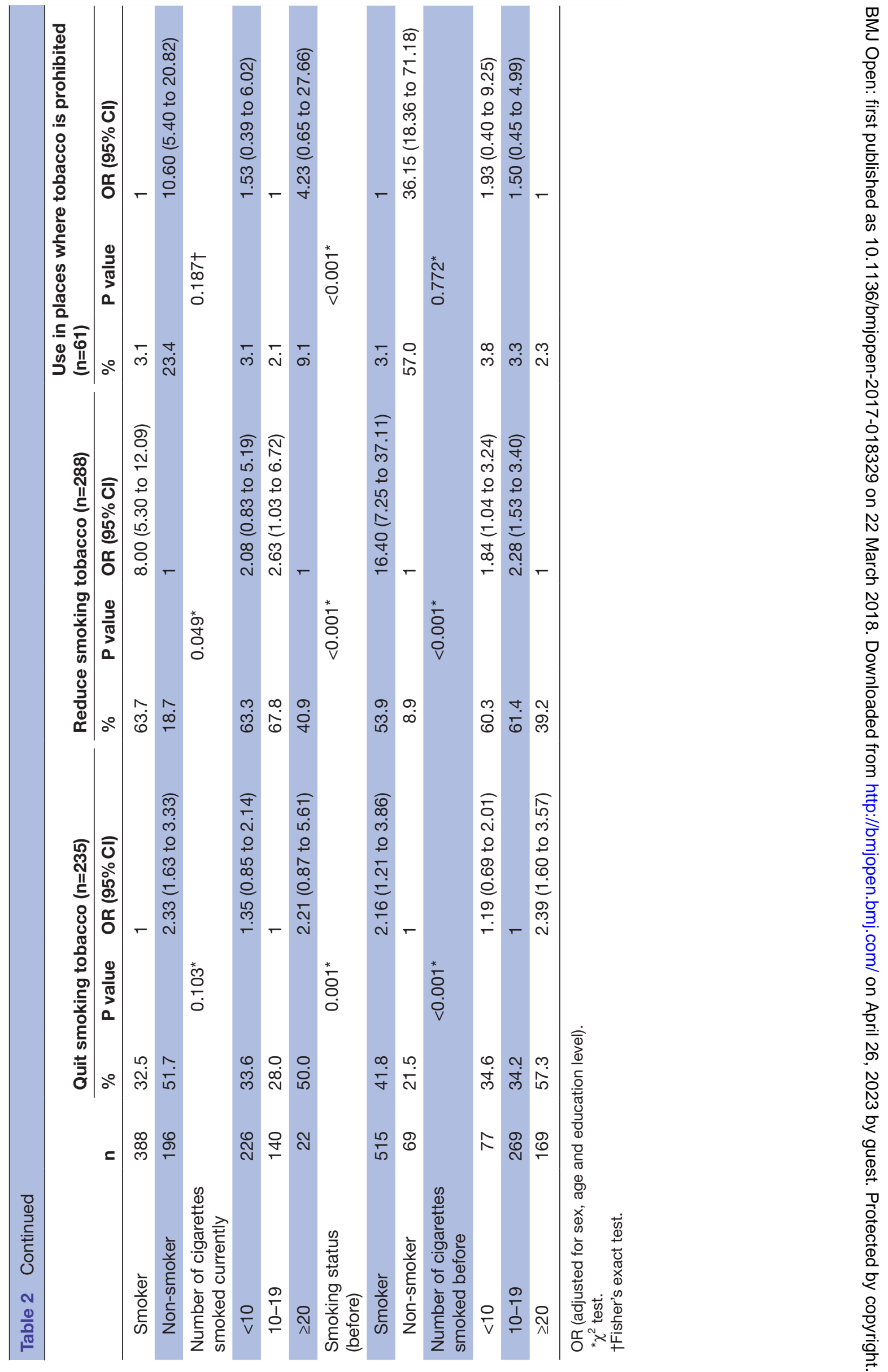




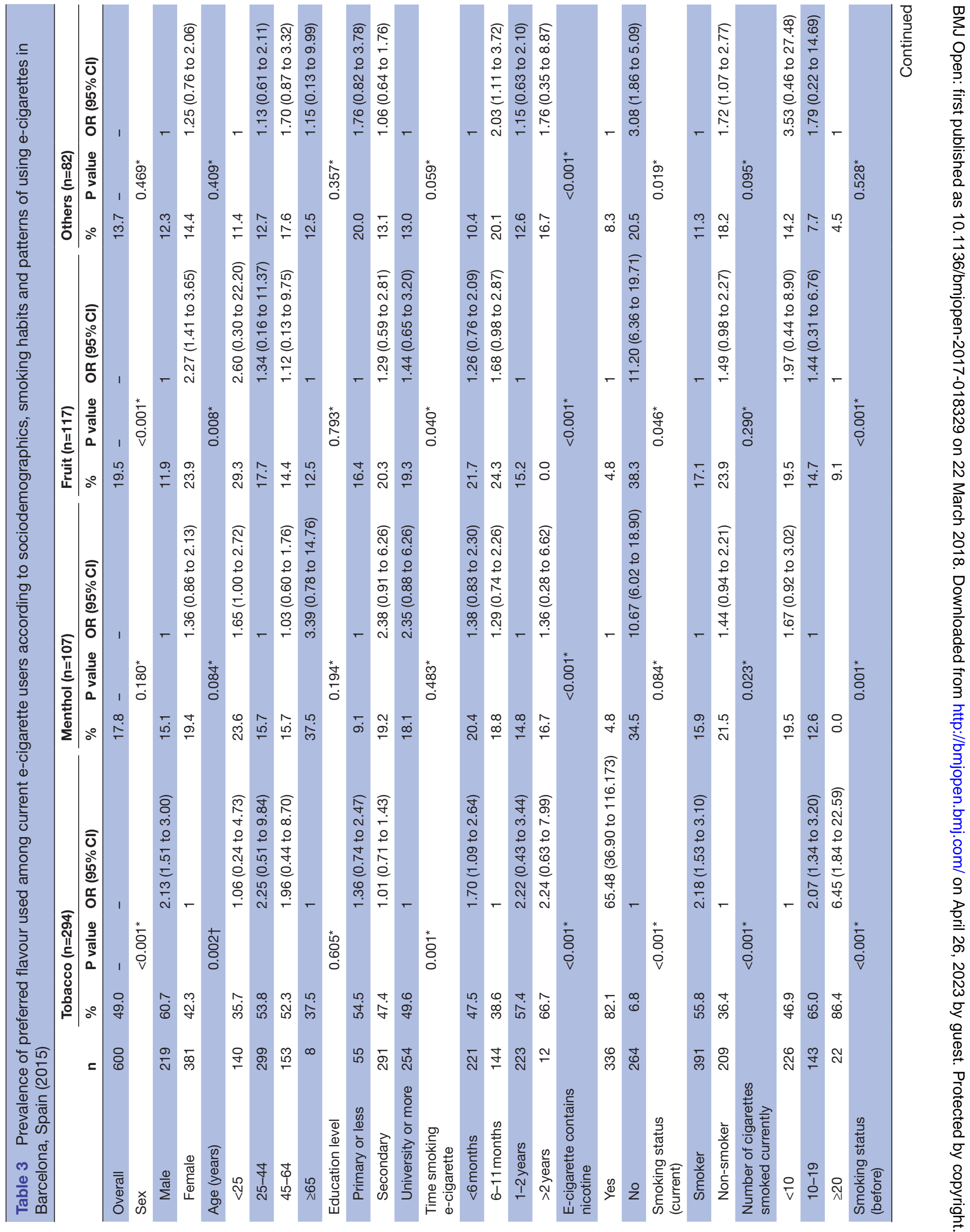




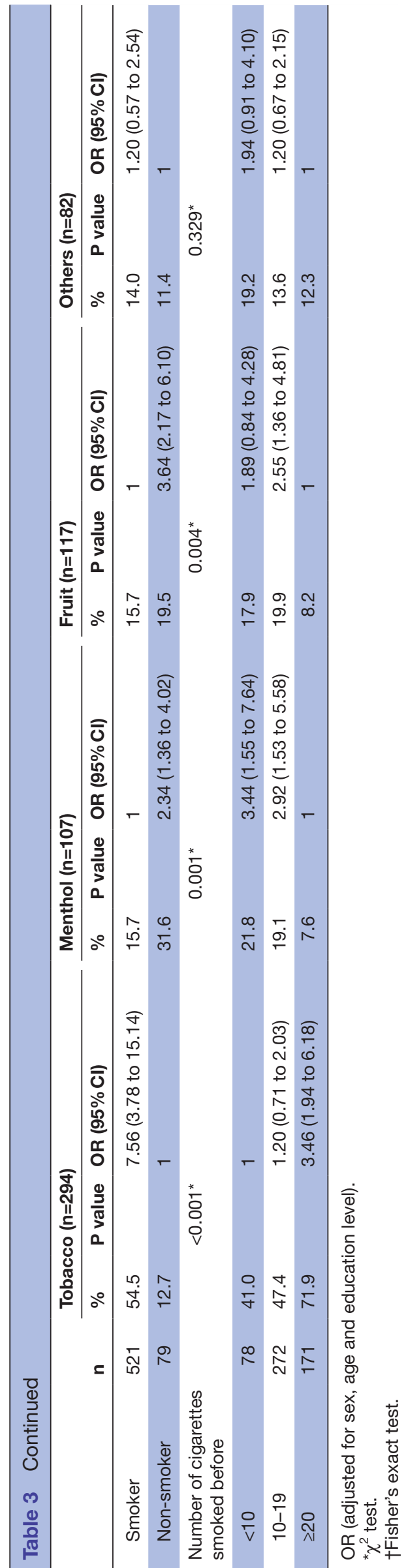

positive. On the other hand, if users continue with dual use they may still be at risk of the negative health outcomes due to tobacco smoking, as even light cigarette smoking can have significant negative health impacts ${ }^{25}$ and a potential risk of long-term health effects of e-cigarette use.

Our data show that men, older people, those who are past and current heavy smokers, and those who use tobacco flavour more frequently were much more likely to use their e-cigarettes with nicotine, suggesting that smoking conventional tobacco is strongly related to use of e-cigarettes with nicotine. However, $16.5 \%$ of participants who were non-smokers before beginning the use of e-cigarettes used liquids with nicotine, indicating a potential for their future nicotine addiction and eventually use of other tobacco products. Conventional tobacco smokers who used e-cigarettes with nicotine reported having reduced the number of cigarettes they smoked at the time of data collection compared with before starting e-cigarette use. This signifies that e-cigarette use with nicotine could contribute to cigarette smokers reducing consumption, which has been supported by past studies, ${ }^{781026}$ but contradicted by others. ${ }^{14}$

Reducing tobacco smoking was the most prevalent motivation for use of e-cigarettes, followed by quitting smoking and to use e-cigarettes in places where tobacco smoking is prohibited. That $90 \%$ of the study participants cited their primary motivation as to quit or reduce tobacco consumption indicates a strong belief that e-cigarettes are effective in helping smokers to do this, which has been documented. ${ }^{27-29}$ A report of e-cigarettes marketing in the UK found that companies often market products as being healthier and safer than traditional cigarettes and effective in cutting down or quitting. ${ }^{30}$

Despite wide bans on flavoured cigarettes, bans on flavoured e-cigarettes are scarce. Research on flavours is necessary to provide evidence on the potential harms of certain e-cigarette flavours to inform related policy decisions. Fifty per cent of our sample preferred tobacco flavour, followed by fruit, menthol and others. Our findings coincide with one study's findings ${ }^{28}$ but differ from other studies which found fruit or menthol to be the preferred flavours, ${ }^{293132}$ and another that found users were more likely to use tobacco flavour at e-cigarette initiation but to switch to other flavours like fruit with continued use. ${ }^{33}$ These differences could be related to the trend of e-cigarette use not having caught on in Spain like it has in countries such as the USA, to differences in restrictions on e-cigarettes marketing or to the relatively short duration of e-cigarette use among our study participants. To our knowledge, no other study has been published that compares preferred flavour of e-cigarettes among individuals' sociodemographic characteristics, patterns of use and tobacco consumption. One comprehensive study from the USA was recently published that assessed preferred non-cigarette tobacco product flavours and stratified by sociodemographic characteristics. ${ }^{32}$ Although the study provided information on the proportion of 
e-cigarette users who use with flavours in general, it did not stratify by the flavours themselves. Our data show that men, heavy smokers and users of e-cigarettes with nicotine were more likely to use tobacco flavour. It makes sense that individuals who use e-cigarettes to use with or replace cigarettes would prefer tobacco flavour. On the other hand, less heavy and non-smokers preferred menthol and fruit flavours. Another study found $80 \%$ of never smokers preferred fruit flavour, $36.4 \%$ preferred menthol and only $6.1 \%$ preferred tobacco. ${ }^{29}$ Fruit and menthol flavours seem to appeal to non-smokers and their availability could be a main reason for non-smokers starting use of e-cigarettes. ${ }^{29}$ Furthermore, fruit flavour may be less effective compared with other flavours in helping cigarette smokers to reduce their tobacco consumption. A recent quasiexperimental study of e-liquid flavour as a moderator of reducing cigarette consumption found that the largest reduction of cigarettes was among the people using menthol flavoured e-cigarettes, while the smallest reduction was among those using chocolate and cherry flavours, even though users of cherry flavour had some of the highest rates of e-cigarette use. ${ }^{31}$

Young people are of particular concern in the discussion surrounding e-cigarettes. A recent study conducted among US high school students ${ }^{16}$ showed that a high concentration of nicotine of e-cigarettes was associated with frequency and intensity of smoking and vaping. Moreover, another study conducted in adolescents in the $\mathrm{UK}^{34}$ showed that the main motivation for using e-cigarettes was for experimentation. In our sample, conducted in adult population, younger people (between 18 and 25 years old) were more often non-smokers before beginning use of e-cigarettes compared with the total sample (25\% and $13 \%$, respectively), suggesting that young people may perceive e-cigarettes as less harmful than conventional cigarettes compared with older people. Furthermore, young people were much more likely to state their main motivation for e-cigarette use as to use them where smoking cigarettes is prohibited. This suggests that they may not be using e-cigarettes as often as older people to reduce the harm of cigarettes, but are instead using them more often for recreation. This suggests young people do not perceive e-cigarettes as being socially stigmatised to the same extent as conventional cigarettes. Young people also use fruit flavoured e-cigarettes at a higher proportion than older people, which was consistent with another study ${ }^{32}$ and also with studies that have been conducted on preferred conventional cigarette flavours. ${ }^{35}$ Studies show that fruity flavours are more appealing to young people, are perceived as being less harmful than tobacco flavour, ${ }^{16}$ and that their availability may be one of the main reasons for young people to experiment with e-cigarettes in the future. ${ }^{29}$

Our study has some limitations. First, we recruited study participants using the method of 'consumer panels', a non-probabilistic sampling technique, which calls into question the external validity of the sample. However, our sample was representative of current e-cigarette users according to sex and age. ${ }^{21}$ Despite our sampling method being a non-probabilistic technique itself, it has been shown in another study to provide greater sample representativeness than other commonly used non-probabilistic sampling methods. ${ }^{21}$ Moreover, previous studies ${ }^{46}$ which we could use to weight our study have small sample sizes and a big limitation of external validity. ${ }^{21}$ For this reason, the data were not weighted. Other limitations are those that exist in studies using surveys as their primary data source. We asked participants to recall information about their past smoking habits, such as the number of cigarettes they smoked before use of e-cigarettes. Therefore, there is a possibility of recall bias or information bias. In this sense, around $30 \%$ of the participants who declared to be non-smokers before use of e-cigarettes also declared used it to quit or reduce tobacco smoking. For this reason, the information collected about users' characteristics before using e-cigarettes should be considered with caution due to the potential for response bias. Moreover, the information about the milligrams of nicotine in the cartridges should be considered with caution, because there are several volumes of e-liquid (from 10 to $30 \mathrm{~mL}$ or more) and the milligrams of nicotine could vary. We also asked users to report their use in places where tobacco smoking is prohibited, including places where e-cigarettes are prohibited and the number of cigarettes they currently smoked. Participants may have under-reported these variables because of social pressures against using e-cigarettes and conventional cigarettes in public places. As a counterpart, we used a face-to-face questionnaire to collect data that provide more internal validity than other methods of data collection such as online questionnaires ${ }^{29}$ or mail-in surveys. ${ }^{37}$ Furthermore, the estimates in stratification with small samples could be considered with caution due to the wide CIs. Moreover, our large sample size of 600 current e-cigarette users adds statistical power to our analyses. Our findings are important, as this is the first study in Spain describing the average time of e-cigarette use, number of cartridges and milligrams of nicotine per cartridge, and the only one in Europe, to our best knowledge, which assesses users' preferred flavour and motivation for use stratified by their patterns of e-cigarette use and past and current tobacco consumption.

In conclusion, according to our results, younger users of e-cigarettes and non-smokers are more likely to use flavours other than tobacco and to use e-cigarettes mainly for recreational purposes. More studies are needed to verify the different theories about e-cigarettes.

Contributors KB drafted the first manuscript with the supervision of JMMS. MF, $M B, E F$ and JMMS contributed to the design and coordination of the study. NMS and CLM prepared the database and KB and JCMS analysed the data. All authors (KB, MF, MB, NMS, CLM, JCMS, EF and JMMS) contributed substantially to the interpretation of data and the successive versions of the manuscript. All authors contributed to the manuscript and approved its final version. JMMS conceived the study and is the principal investigator of the project.

Funding The project was cofunded by the Instituto de Salud Carlos III, Subdirección General de Evaluación, Government of Spain (PI15/00291). The project was also cofunded by ISCIII-Subdirección General de Evaluación and by FEDER funds/European Regional Development Fund (ERDF) — a way to build Europe - in 2015 and Ministry of Universities and Research, Government of Catalonia (grant 2017SGR608). 
Competing interests None declared.

Patient consent Obtained.

Ethics approval Ethics Committee of the Bellvitge University Hospital

Provenance and peer review Not commissioned; externally peer reviewed.

Data sharing statement № additional data are available.

Open Access This is an Open Access article distributed in accordance with the Creative Commons Attribution Non Commercial (CC BY-NC 4.0) license, which permits others to distribute, remix, adapt, build upon this work non-commercially, and license their derivative works on different terms, provided the original work is properly cited and the use is non-commercial. See: http://creativecommons.org/ licenses/by-nc/4.0/

(c) Article author(s) (or their employer(s) unless otherwise stated in the text of the article) 2018. All rights reserved. No commercial use is permitted unless otherwise expressly granted.

\section{REFERENCES}

1. King BA, Patel R, Nguyen $\mathrm{KH}$, et al. Trends in awareness and use of electronic cigarettes among US adults, 2010-2013. Nicotine Tob Res 2015;17:219-27.

2. Hajek P, Etter JF, Benowitz N, et al. Electronic cigarettes: review of use, content, safety, effects on smokers and potential for harm and benefit. Addiction 2014;109:1801-10.

3. Dockrell M, Morrison R, Bauld L, et al. E-cigarettes: prevalence and attitudes in Great Britain. Nicotine Tob Res 2013;15:1737-44.

4. Martínez-Sánchez JM, Ballbè M, Fu M, et al. Electronic cigarette use among adult population: a cross-sectional study in Barcelona, Spain (2013-2014). BMJ Open 2014;4:e005894.

5. Faculty of Public Health. Nicotine Science and Policy, Letter of concern by 53 public health experts to $\mathrm{WHO}$ with regard to the FCTC and electronic cigarettes. http://nicotinepolicy.net/documents/letters/ MargaretChan.pdf (accessed 4 May 2017).

6. Centre for Tobacco Control, Research and Education. Letter of support from 129 public health experts for WHO's evidence based approach to electronic cigarettes. https://tobacco.ucsf.edu/sites/ tobacco.ucsf.edu/files/u9/Chan-letter-June16\%20PST\%20FINAL\% 20with\%20129\%20sigs.pdf (accessed 4 May 2017).

7. Rahman MA, Hann N, Wilson A, et al. E-cigarettes and smoking cessation: evidence from a systematic review and meta-analysis. PLoS One 2015;10:e0122544.

8. Khoudigian S, Devji T, Lytvyn L, et al. The efficacy and short-term effects of electronic cigarettes as a method for smoking cessation: a systematic review and a meta-analysis. Int $J$ Public Health 2016;61:257-67.

9. McRobbie H, Bullen C, Hartmann-Boyce J, et al. Electronic cigarettes for smoking cessation and reduction. Cochrane Database Syst Rev 2014:CD010216.

10. Brown J, Beard E, Kotz D, et al. Real-world effectiveness of e-cigarettes when used to aid smoking cessation: a cross-sectional population study. Addiction 2014;109:1531-40.

11. Goniewicz ML, Gawron M, Smith DM, et al. Exposure to Nicotine and Selected Toxicants in Cigarette Smokers Who Switched to Electronic Cigarettes: A Longitudinal Within-Subjects Observational Study. Nicotine Tob Res 2017;19:160-7.

12. Polosa R, Morjaria JB, Caponnetto P, et al. Persisting long term benefits of smoking abstinence and reduction in asthmatic smokers who have switched to electronic cigarettes. Discov Med 2016;21:99-108.

13. Cibella F, Campagna D, Caponnetto P, et al. Lung function and respiratory symptoms in a randomized smoking cessation trial of electronic cigarettes. Clin Sci 2016;130:1929-37.

14. Kalkhoran S, Glantz SA. E-cigarettes and smoking cessation in realworld and clinical settings: a systematic review and meta-analysis. Lancet Respir Med 2016;4:116-28.
15. WHO Study Group on Tobacco Production Regulation. WHO Study Group on Tobacco Product Regulation. Report on the scientific basis of tobacco product regulation: third report of a WHO Study Group. World Health Organ Tech Rep Ser 2009:1-41.

16. Goldenson NI, Leventhal AM, Stone MD, et al. Associations of Electronic Cigarette Nicotine Concentration With Subsequent Cigarette Smoking and Vaping Levels in Adolescents. JAMA Pediatr 2017;171:1192-9.

17. Pisinger $C$, Døssing M. A systematic review of health effects of electronic cigarettes. Prev Med 2014;69:248-60.

18. Behar RZ, Davis B, Wang Y, et al. Identification of toxicants in cinnamon-flavored electronic cigarette refill fluids. Toxicol In Vitro 2014;28:198-208.

19. Kim HJ, Shin HS. Determination of tobacco-specific nitrosamines in replacement liquids of electronic cigarettes by liquid chromatography-tandem mass spectrometry. J Chromatogr A 2013;1291:48-55.

20. Kosmider L, Sobczak A, Fik M, et al. Carbonyl compounds in electronic cigarette vapors: effects of nicotine solvent and battery output voltage. Nicotine Tob Res 2014;16:1319-26.

21. Matilla-Santander N, Fu M, Ballbè M, et al. [Using consumer panels in public health observational studies]. Gac Sanit 2017;31.

22. Kozlowski LT, Porter CQ, Orleans CT, et al. Predicting smoking cessation with self-reported measures of nicotine dependence: FTQ, FTND, and HSI. Drug Alcohol Depend 1994;34:211-6.

23. Farsalinos KE, Romagna G, Tsiapras D, et al. Characteristics, perceived side effects and benefits of electronic cigarette use: a worldwide survey of more than 19,000 consumers. Int J Environ Res Public Health 2014;11:4356-73.

24. Pepper JK, Ribisl KM, Emery SL, et al. Reasons for starting and stopping electronic cigarette use. Int J Environ Res Public Health 2014;11:10345-61.

25. Lechner WV, Tackett AP, Grant DM, et al. Effects of duration of electronic cigarette use. Nicotine Tob Res 2015;17:180-5.

26. Hartmann-Boyce J, McRobbie H, Bullen C, et al. Electronic cigarettes for smoking cessation. Cochrane Database Syst Rev 2016;9.

27. Etter JF, Bullen C. Electronic cigarette: users profile, utilization, satisfaction and perceived efficacy. Addiction 2011;106:2017-28.

28. Dawkins L, Turner J, Roberts A, et al. 'Vaping' profiles and preferences: an online survey of electronic cigarette users. Addiction 2013;108:1115-25.

29. Berg CJ. Preferred flavors and reasons for e-cigarette use and discontinued use among never, current, and former smokers. Int $J$ Public Health 2016;61:225-36.

30. De Andrade M, Hastings G, Angus K, et al. The marketing of electronic cigarettes in the UK. Cancer Research UK 2013 https:// www.cancerresearchuk.org/sites/default/files/cruk_marketing_of_ electronic_cigs_nov_2013.pdf (accessed 2 May 2017).

31. Litt MD, Duffy V, Oncken C. Cigarette smoking and electronic cigarette vaping patterns as a function of e-cigarette flavourings. Tob Control 2016;25:ii67-ii72.

32. Bonhomme MG, Holder-Hayes E, Ambrose BK, et al. Flavoured noncigarette tobacco product use among US adults: 2013-2014. Tob Control 2016;25:ii4-ii13.

33. Farsalinos KE, Romagna G, Tsiapras D, et al. Impact of flavour variability on electronic cigarette use experience: an internet survey. Int J Environ Res Public Health 2013;10:7272-82.

34. Bauld L, MacKintosh AM, Eastwood B, et al. Young People's Use of E-Cigarettes across the United Kingdom: Findings from Five Surveys 2015-2017. Int J Environ Res Public Health 2017;14:973.

35. Klein SM, Giovino GA, Barker DC, et al. Use of flavored cigarettes among older adolescent and adult smokers: United States, 20042005. Nicotine Tob Res 2008;10:1209-14.

36. Lidón-Moyano C, Martínez-Sánchez JM, Fu M, et al. [Prevalence and user profile of electronic cigarettes in Spain (2014)]. Gac Sanit 2016;30:432-7.

37. Biener L, Song E, Sutfin EL, et al. Electronic cigarette trial and use among young adults: reasons for trial and cessation of vaping. Int $J$ Environ Res Public Health 2015;12:16019-26. 